\title{
La importancia sociohistorica y cultural de las revistas de la Universidad Nacional
}

Socio-historial and cultural relevance of the journals of Universidad Nacional

\section{A importância sócio-histórica e cultural dos periódicos da Universidade Nacional.}

Marybel Soto-Ramírez

Universidad Nacional

Costa Rica

Recibido: 15/04/2021 - Aprobado: 20/05/2021

\begin{abstract}
Resumen
El ensayo plantea que las revistas académicas cumplen un papel sociohistórico y cultural decisivo que abona en la labor de la universidad como institución de cultura. Para ello propone un recorrido sobre algunos de los hitos de la constitución de la prensa periódica científica de la Universidad Nacional y abunda en el papel histórico y nodal de la revista como práctica de orden eminentemente académico o intelectual en la comunicación de la ciencia.
\end{abstract}

Palabras clave: revistas académicas, estudios sobre edición universitaria, Editorial Universitaria, pensamiento latinoamericano

Abstract

The essay states that journals play a decisive socio-historical and cultural role that contributes to the work of the university as an institution of culture. To this end, it proposes a journey through some of the milestones of the constitution of the scientific periodical press of the National University and elaborates on the historical and pivotal role of the journal as an eminently academic or intellectual practice in the communication of science.

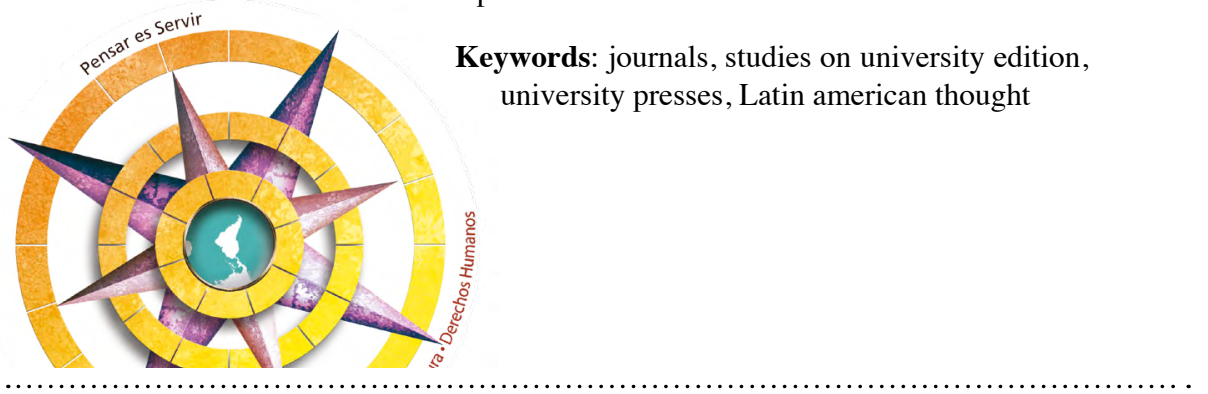

Doi: https://doi.org/10.15359/tdna.37-70.2 


\section{Resumo}

$\mathrm{O}$ ensaio argumenta que as revistas académicas desempenham um papel sócio-histórico e cultural decisivo que contribui para o trabalho da universidade como instituição de cultura. Para tal, propõe uma visita guiada a alguns dos marcos da constituição da imprensa periódica científica da Universidade Nacional e abunda no papel histórico e nodal da revista como prática de ordem eminentemente académica ou intelectual na comunicação da ciência.

Palavras chave: periódicos acadêmicos, estudos sobre publicações universitárias, publicações universitárias, pensamento latino-americano

En diversas oportunidades, y ante el avance tan valioso e importante que ha tenido la publicación periódica en la Universidad Nacional, Costa Rica, los cónclaves de especialistas han debatido sobre el papel que corresponde a la revista académica en el conglomerado de la difusión de la ciencia. Esto es, sin duda, un aspecto medular para orientar las políticas de divulgación del conocimiento. La reflexión se ha orientado a que, como universidad pública, debemos responder a los procesos de democratización en el acceso al conocimiento en atención, no solo a la comunidad académica donde nos desarrollamos, sino también a la sociedad como un todo que nos sustenta. La publicación periódica cumple un papel decisivo en esta relación.

Del estudio de las revistas, de la prensa periódica, se logra extrapolar información vital en las investigaciones especializadas sobre ellas o en ellas, dependiendo del prisma que rija nuestro posicionamiento, en tanto fuentes y objetos de estudio. Si bien la práctica investigativa sobre revistas ya tiene algunos años, despuntando principalmente con los estudios de revistas culturales icónicas como SUR, CARAS Y CARETAS, CUADERNOS AMERICANOS, REPERTORIO AMERICANO, el avance en los desarrollos metodológicos y la construcción misma de los objetos de estudio han permitido ampliar espectros hacia otras revistas, como las las científicas, o incluso hacia impresos que podrían parecernos nimios, como las denominadas "revistas del corazón", cuyo estudio arroja muy interesantes resultados sobre conformación de campos culturales y la relación con las culturas populares. Es importante anotar asimismo, más recientemente, los análisis sobre los

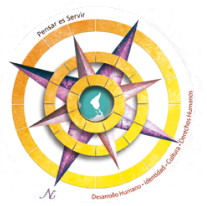


tipos de comunicación en la era digital, como los blogs y la conformación de redes semánticas.

La investigación "revisteril", donde se analizan formatos, soportes, presencias, participaciones, consignas y la importante temática de constitución de redes, son todos ellos temas susceptibles de análisis en los estudios sobre edición universitaria, en general y en particular, en la edición de revistas académicas. Por ello, es importante concordar en que la concepción y conducción de una revista, es ante todo, una tarea de orden intelectual, inserta en la actividad de la cultura letrada. Es por ello que Beatriz Sarlo (1992) señala en su artículo, "Las razones de una práctica", que ante el llamado o declaración de un grupo de "Publiquemos una revista" (p.1) subyace un sentido plural, que engloba, en primera instancia, un nosotros, los productores de ese medio, y segundo, comprende una intervención, en este caso de orden cultural. Sarlo apunta que toda revista pone el acento sobre lo público y está en tiempo presente, para la audiencia contemporánea. De hecho, por estas características, la revista como objeto de estudio, es un testimonio de una coyuntura. Esta condición es lo que las hace tan valiosas para comprender formas de circulación $\mathrm{y}$ de producción de contenidos e ideas, de ellas en sí mismas como objetos culturales y de su lugar en la recepción de ideas.

Considero que han sido menos las oportunidades que como universidad hemos tenido de reflexionar sobre este papel sociohistórico y cultural que cumple la publicación periódica científica, como fuente de estudio y en su condición de objeto de difusión de la ciencia. Esta segunda precisión es importante, porque tal como lo han señalado Beatriz Sarlo (1992), Alexandra Pita (2005) y Mario Oliva (2009), entre otros estudiosos, es en las revistas donde se engarza una cierta forma de organización de conocimiento o, más ampliamente, de participación intelectual, que da cuenta de los proyectos políticos, culturales, educativos o de divulgación a los que como institución hemos aspirado.

Apunta Sarlo (1992) que las revistas son testimonio de una periodización, de las problemáticas, de los esfuerzos, de los puntos de atención epistemológica o estética de un momento dado. (1992, p. 1). Puede corroborarse lo interesante 
de esta propuesta de la teórica argentina, porque señala que "la modernización tuvo en las revistas su instrumento; y los debates tienen su arena en las revistas" (p. 12).

Quisiera centrar mi propuesta en un punto que considero valioso, pertinente y necesario cuando se realiza investigación sobre la prensa periódica universitaria; tal es el de la memoria histórica y la importancia de la revista académica desde una visión de historia intelectual y de análisis revistológico que le asiste. Recurrir a la memoria histórica nos permite mirar de dónde venimos y fijar los derroteros y rumbos que mejor respondan a las exigencias de los tiempos, en nuestro caso, a las exigencias de la universidad pública latinoamericana y de las formas de comunicar la ciencia, es decir, la práctica de los lenguajes en uso. Es, por tanto, un posicionamiento situado.

Según las actas de la comisión adhoc que organiza la Universidad Nacional de Costa Rica, desde el 2 de abril de 1974, se planteó la necesidad de la publicación de una revista como parte de los esfuerzos político-educativos en que se fundamentaba la nueva casa de estudios. La publicación de esa revista apuntaba a una cuyo nombre había puesto a Costa Rica, a inicios del siglo XX, en el mapa cultural latinoamericano, durante casi 40 años de existencia. Repertorio Americano es la primera revista que decidió publicar la naciente Universidad, según se establece en el Acta 93, de la Comisión Ad-hoc, en una propuesta gestionada fuertemente por el entonces Ministro de Trabajo, Lic. Francisco Morales, ante los miembros de la Comisión. La Universidad Nacional logró concretar aquella propuesta, considerada por el Lic. Morales como deuda pendiente con el Benemérito de la Patria, Joaquín García Monge, a partir de dos vertientes: una, contar con una colección completa de los 1158 números que componen la edición de Repertorio Americano de 1919 a 1958; segundo, realizar la negociación legal por los derechos de uso del nombre de la revista ante los herederos de la familia de García Monge. Considero que con estas acciones la UNA respondería tempranamente a ese llamado intelectual que nos apuntaba Beatriz Sarlo, al inicio de este ensayo, de publiquemos una revista y lo asume como un deber de su condición de institución académica.

Efectivamente, lo que se conoce como la tercera salida de Repertorio Americano, a saber, la publicación a partir de 1974, correspondía a otra revista, no era la magnífica

4 La importancia sociohistorica y cultural de las revistas de la

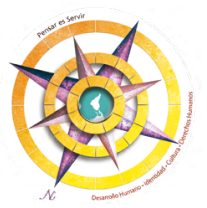


publicación gestionada por García Monge, sino que se trataba de una edición que surgía inserta en el quehacer intelectual y académico de la naciente universidad, es decir, se trata de una revista universitaria. ¿Por qué puede ser importante esta referencia a momentos fundacionales de la Universidad cuando se analiza la relación con las revistas y sus desafíos? Precisamente porque nos permite sopesar la importancia vital que la UNA, concedió a la necesidad de publicación de revistas que permitieran, como diría en setiembre de 1974, el Dr. Chéster Zelaya, miembro de dicha Comisión: "ofrecerlas como instrumento de canje" por ejemplo, y añadiría, refiriéndose expresamente al caso de Repertorio Americano: "Ya hemos recibido respuesta de Alemania, Italia, Francia, etc." en su interés por establecer los canjes interbibliotecarios. El Dr. Zelaya informaba al Rector Núñez al justificar la importancia de los impresos: "Es un gran negocio con esos cambios porque son revistas de gran valor académico" que aportarán positivamente para la constitución de acervos (Acta 124). Casi al mismo tiempo que Repertorio Americano, también se fundarían otras revistas institucionales especializadas, como la Revista Geográfica de América Central, posteriormente Praxis, de la Escuela de Filosofía, y la Revista de
Historia, en 1975, luego Letras de la Escuela de Literatura y la Revista de Ciencias Veterinarias, en 1979.

Desde su inicio, las revistas fueron concebidas como instrumentos de canje, como medios de difusión especializados y especiales para dar a conocer la producción académica y como formas de demostrar la madurez de la profesionalización en los distintos ámbitos de formación académica. Por eso es interesante hacer referencia lo que indicaba en 1979 , el Rector, Dr. Alfio Piba, cuando declaraba sobre la repercusión que tendría una revista de ciencias veterinarias para el país y para la difusión del pensamiento científico, pues serviría: “... para elevar con ella el nivel cultural científico técnico de todos los profesionales que comprenden el área de influencia de Costa Rica" (Ciencias Veterinarias, No. 1, sept. 1979)

Un segundo aspecto importante de tomar en consideración es que la publicación periódica científica se considera como un lieú, un lugar-espacio, para la reunión de los materiales académicos resultantes de las diversas áreas del quehacer y, según abundaba el Dr. Piba, para que dichos materiales producidos por el profesorado no quedasen desvinculados en publicaciones quizá poco serias, sino que, por el contrario, la 
Universidad asegurara un soporte de calidad para las publicaciones de sus jóvenes catedráticos, en revistas que ella misma publicara.

Es valioso reiterar que la Universidad Nacional, desde su nacimiento, comprendió el valor intrínseco y definitivo de la publicación periódica, no solo para la divulgación y difusión del conocimiento sino como un hecho de valor en sí mismo a que estaba llamada como universidad y por tanto, institución de cultura.

Es importante señalar asimismo que las revistas establecen una relación temprana con la Editorial Universidad Nacional, de igual forma en proceso de conformación y consolidación, para la gestión de la impresión en lo que se denominaba, entonces, como el Taller de Publicaciones e Impresiones, hoy Departamento de Publicaciones, y, en algunos casos, también por medios externos, mediante licitación, cuando se realizaban traslados de presupuesto o se lograban apoyos de instituciones externas para realizar la impresión de los números. En el caso de Repertorio Americano recibió durante algunos años, un estipendio de la Caja Costarricense del Seguro Social para impresión de números; otro ejemplo de este tipo de relación la tuvo la Revista de Historia. La búsqueda de presupuestos externos que coadyuvaran a las escasas rentas de la Editorial y de la Universidad, fue una situación normal y constante en los esfuerzos de publicación de las revistas cuyos costos de producción recaía en las unidades académicas donde estaban adscritas.

La mayoría de revistas durante este período no estuvieron formalmente conceptualizadas como proyectos, sino que se asumían dentro de la carga académica del profesorado. Posiblemente por ello, en algunos casos, hubo poca continuidad en estilo, en calidad y en la periodicidad de ellas. Durante la década de 1980, la preocupación del Consejo Editorial fue constante sobre la permanencia y la calidad de las revistas; respecto de las cuales incluso los mismos representantes de las facultades señalaban que debían de pasar los "filtros de calidad y responder como propuestas constituidas de verdaderos órganos de expresión científica, literaria o artística, nacional o ístmica y de carácter estrictamente universitario" siendo avaladas por esferas de decisión más que por "grupos entusiastas de escuelas, centros o institutos". (EUNA, 1982).

En 1981 se normaron los requisitos básicos que debían expresar las revistas de la EUNA: establecer en sus

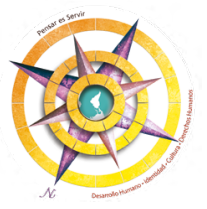


primeras páginas el propósito, el por qué se había fundado, a quiénes iba dirigida, la periodización, la mención expresa a las autoridades de la universidad, al decano y director de centro o escuela, a los integrantes del comité de redacción --entonces no se denominaba comité editorial-- y al director de la revista; debía asimismo incluir expresamente la solicitud de canje, la prohibición de reproducción total o parcial sin autorización previa y explícita del director de la publicación, y, un dato muy interesante: que todos los artículos debían contener un resumen con su correspondiente traducción al inglés (abstract) y en lo posible "al francés o portugués (resumé-resumo); así como las condiciones de las colaboraciones; la no devolución de originales, la entrega de 10 separatas a los autores y un ejemplar de la revista y el valor de las suscripciones y número suelto (Acta 19-81, 31 de agosto 1981). Con lo aquí establecido es posible aducir que ya en la década de 1980 existía claridad en el sentido de modernidad que se quería imprimir a las revistas desde la EUNA.

Con el pasar de los años, otras revistas fueron naciendo en las escuelas y también en las facultades como la Revista $A B R A$, en Ciencias Sociales e ISTMICA, en la Filosofía y Letras, hasta ir conformando el panorama de la edición actual de la prensa periódica científica institucional.

En el quinquenio 2005, bajo la rectoría del Dr. Olman Segura Bonilla, la Vicerrectora de Extensión, Dra. Elizabeth Ramírez, plantearía la importancia que desde la administración superior se fijó en el mejoramiento de la calidad de las revistas institucionales. La búsqueda de definición de un nombre que las incluyera a todas en sus diversidades, llevó a denominarles revistas institucionales para reforzar el sentido de que eran producto y expresión de ese quehacer universitario en contraste con lo que en algunos casos se acusaba en algunas de ellas de haberse convertido en "proyectos personales".

Desde 2005 la Vicerrectoría de Extensión puso denodado interés en solventar el que se identificó como el problema principal de las revistas: la falta de periodicidad y las deudas que sostenían con el Departamento de Publicaciones por el pago de impresión de las mismas. En algunos casos, por ejemplo Repertorio Americano, se presupuestaba en las cuentas de operación la compra de papel desde el Instituto de Estudios Latinoamericanos, instancia a la que pertenece dicha revista, y se trasladaba ese insumo al Departamento de Publicaciones para 
su utilizarse durante el año en la impresión de la revista.

Como política de la Vicerrectoría de Extensión en dicho período se convocaron varios talleres con las personas académicas encargadas de las revistas para definir cuáles eran las necesidades, y principales carencias, que afrontaban en su quehacer cotidiano. Se atendieron requerimientos de mobiliario, equipo de computo, tintas de impresora, entre otros. Casi todas las personas responsables de revistas señalaban que la falta de financiamiento, las deudas pendientes en el Departamento de Publicaciones e Impresiones, así como la necesidad de capacitación, eran sus problemáticas más urgentes.

En consecuencia, desde la administración del Dr. Segura Bonilla y la Dra. Ramírez, se definió un plan que buscó, en primera instancia, regularizar las revistas bajo las siguientes condiciones:

1. Que todas las revistas se constituyeran como proyectos académicos. Algunas ya lo eran desde su nacimiento, por ejemplo $R e$ pertorio Americano y posteriormente Temas de Nuestra Améri$c a$, la cual salió a la luz en 1983 , habían nacido como actividades académicas permanentes.
2. Que todas las revistas avanzaran hacia la consecución del sello editorial EUNA, de tal forma que se convirtieran en productos editoriales plenos, con clara identificación de su pertenencia a la UNA, --aunque desde 1981 se hablaba en actas de las revistas de la EUNA-- y su financiamiento pasara a dicha instancia

3. Que todas iniciaran procesos de indexación, para lo cual, los requisitos para el logro del sello editorial EUNA, lineamiento que se emitió desde Consejo Editorial, se alinearon con los requisitos de LATINDEX, de tal forma que, casi de manera inmediata cuando una revista obtuviera el sello editorial también cumpliera con los criterios mínimos para someterse a evaluación de LATINDEX

4. Que se iniciara la investigación sobre las revistas electrónicas y sus posibilidades de avanzar hacia ese soporte. Había un interés muy enfocado en lograr que las revistas migraran a digitales. Esta propuesta fue guía del rumbo la gestión de la Dra. Ramírez.

Desde la administración del Rector Segura Bonilla se fortaleció a la Editorial presupuestariamente. Se

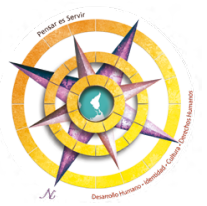


asumió y saldó las deudas pendientes de las revistas con el Departamento de Publicaciones y se puso en marcha un proceso integral para el fortalecimiento de las revistas de forma coordinada entre la EUNA y la Vicerrectoría de Extensión. Con la estrategia, "Fortalecimiento de las capacidades de las revistas institucionales" una serie de talleres, coloquios y lineamientos que buscaban dicho objetivo, se propuso el logro de las siguientes metas:

1. la incorporación de las revistas como proyectos de gestión académica al formularlas como tales;

2. la investigación para avanzar hacia la revista digital, que en ese momento aún era poco viable por falta de investigación y pruebas con software especializado para edición

3. sensibilizar sobre la resistencia al cambio del soporte impreso al digital y los elementos de indexación.

4. establecer que todas las revistas se acogieran evaluación para tener el sello editorial

Para el siguiente quinquenio, a partir del 2009, se continuó con el apoyo a los procesos de fortalecimiento pero esta vez mucho más centrado en $l a$ gestión académica de las revistas.

El reto que se fijó la administración de la Rectora Sandra León Coto, fue de que todas las entonces denominadas, revistas institucionales, lograran tener el sello editorial, lograran indexación y se "virtualizaran", reconociendo en el soporte digital mayores ámbitos de difusión, menores tiempos de edición y costos de producción y mayor nivel de circulación y acceso a ellas.

El criterio de contar con el sello editorial y el de la formulación de la revista como proyecto académico de gestión, definirían el siguiente paso, la denominada "virtualización", que era en realidad, la publicación de las revistas en nuevo soporte, pasando así, del impreso al digital.

Bajo la dirección del Dr. Mario Oliva, en la Vicerrectoría de Extensión, un nuevo impulso catapultaría a las revistas académicas hacia su fortalecimiento estrechamente vinculado con la EUNA y la Dirección de Tecnologías de Información, específicamente, con la sección de UNAWEB. Gracias a un sistemático y riguroso proceso de investigación, análisis y adecuación a las necesidades de la Universidad Nacional, el desarrollo del Portal de Revistas Institucionales 
y la adopción del Open Journal System como gestor dieron norte a un proceso que fue revolucionario. Mediante talleres conjuntos entre la Vicerrectoría de Extensión, la Editorial Universidad Nacional y UNAWEB, se realizaron pruebas piloto con varias revistas para lograr este propósito de cambio al soporte digital. Participaron en dicho programa piloto, la Revista de Ciencias Geográfica, Ensayos Pedagógicos, ABRA, ISTMICA, Temas de Nuestra América y REVMAR entre otras. Con la idea de hacer un "diseño específico" del gestor acorde con las necesidades de la UNA, se inició una árdua tarea de investigación, diálogo e implementación, labor que realizaron de manera excelente los ingenieros informáticos de UNAWEB y de lo cual daban cuenta en comunicaciones oficiales del año 2011 cuando señalaban "Para celebrar la exitosa conclusión de este proyecto, la DTIC, la EUNA y la Dirección de Extensión invitaban al lanzamiento oficial del portal el 31 de agosto de dicho año" (Carta Oficial DTIC)

La asignación de presupuestos para las necesidades de las revistas se hizo práctica común, establecida desde la Vicerrectoría de Extensión, mediante el traslado presupuestario para adquisición de activos y, por parte de la EUNA, se continuó sufragando gastos de producción de las revistas.

Posteriormente, tanto las Vicerrectorías de Investigación y de Extensión articularían el Programa de mejoramiento de calidad de la publicación académica de las revistas universitarias, con apoyo del CONARE, y sería en el año 2014 cuando se apruebó la creación de la Subcomisión de Revistas y Repositorios, con carácter permanente.

Los planes de capacitación anuales de directores de revistas, los procesos de capacitación en los requisitos para indexación, el diseño, elaboración y puesta en marcha del gestor OJS, la creación del portal de revistas institucionales organizado de manera tripartita UNAWEB-EXTENSIÓN y la EUNA, hecho consignado en la imagen gráfica del portal, y la consecuente producción y circulación de revistas en soporte digital y la promoción de migración de estas a formato electrónico, fueron hitos en este período. Para que una revista pudiera estar presente en el portal, se requería que tuviera el sello editorial y que estuviera constituida como proyecto en el área de extensión.

Asímismo, las acciones desarrolladas en este período en coordinación entre la EUNA y la Vicerrectoría

10 La importancia sociohistorica y cultural de las revistas de la

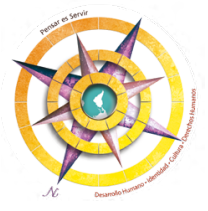


de Extensión para brindar apoyo a las revistas en traducción, filología, diagramación, equipos de computo y sobre todo, el diseño de la Propuesta de lineamientos, criterios y procedimientos para la gestión académica de revistas, documento elaborado por las asesoras académicas, MSc. Marlene Flores Abogabir y MDDHH María Eugenia Restrepo, con participación de funcionarios de la Editorial y validación por los coordinadores de las revistas, fijó un valioso instrumento de trabajo y funcionamiento consensuado que incluía en un flujo de proceso que unía a todos los participantes: instancia académica, revista, direcciones de áreas, es decir, vicerrectorías, EUNA, UnaWeb, y Departamento de Publicaciones, para avanzar en este objetivo de fortalecer la publicación periódica en la UNA, mediante procesos más expeditos de publicación.

El trabajo fija los derroteros de la internacionalización, tanto por el portal de la UNA, como por el portal de portales de CONARE, KIMUK, los apoyos a los marcajes para SCIELO, en 2014, para EDUCARE, como primera revista electrónica de la UNA, la adopción del DOI, a partir de 2015 y posteriormente la inclusión de Costa Rica en La Referencia.
El presente es un recorrido somero, pero puede corroborarse que las revistas de la Universidad Nacional han respondido en el cumplimiento de su misión y vocación histórica, la difusión cultural de la universidad hacia la comunidad, la difusión académico-científica que por definición y antonomasia les corresponde y la aspiración que desde 1974, con las primeros órganos de divulgación académica que se fundan, se ansiaba lograr: el de tener una interacción permanente Universidad-comunidad académica y sociedad.

Las revistas, al igual que los libros, están a la base de la universidad como institución de cultura, como representación de la ciudad letrada, en la diseminación del conocimiento científico, en el fungir como esos repositorios de saber, de políticas de investigación y de temas de interés en un momento dado, de la formación de pensamiento crítico y sobre todo, de la promoción del diálogo entre pares y con la comunidad que nos sustenta.

La profusión de actores que encierra el verbo en plural al que hacía referencia Beatriz Sarlo en su enunciado de Publiquemos una revis$t a$, no es algo fácil, ni dado, ni que pueda recaer en una sola persona o en una sola instancia. Implica una 
conjunción de voluntades: las del rigor científico de los artículos que se publican, en primera instancia, la seriedad de los pares que evalúan, la labor de detalle de editores, directores y comités editoriales, y el necesario enramado que la prensa universitaria que, junto con las vicerrectorías, se entretejen para crear un sistema de difusión del conocimiento.

Por ello, afirmo que el valor sociohistórico y cultural de estos órganos de difusión académica, ante el llamado de Publiquemos una revista, está en la suma de voluntades para hacer ese hecho, revolucionario en todo aspecto, algo posible.

La permanencia de las revistas en el tiempo --y su estudio-- nos permiten aquilatar el precioso aporte de esa conjunción de voluntades para dar cuenta de un estado del avance del conocimiento en un momento y lugar dados. Pero también da cuenta de un hecho político en el proyecto educativo de la universidad que marca el que se publicaran ciertas revistas, ciertos artículos, cierta cantidad de páginas, ciertos temas e, incluso, ciertos autores. Todo estos aspectos son susceptibles de investigación.
De ahí que sea tan vital también para quienes estudiamos la revista como objeto en la construcción cultural, que estos "almacenes de un tiempo y un conocimiento", como señala Ribler Pika (2014) se mantengan accesibles y que aún si bien estamos en épocas donde lo digital es símbolo de los tiempos, se valore la posibilidad también de mantener, aunque sea para los estudiosos, un pequeño acervo en físico de las ediciones, como instrumentos clave para el análisis de estas tensiones, de los desarrollos y de los aciertos en algo que es crucial y que ya amerita de nuestra atención: la formulación de una historia de las revistas institucionales de la Universidad Nacional, en su doble papel, como proyecto académico y como proyecto editorial, dentro de un proyecto mayor que es el político-educativo de nuestra Universidad Nacional.

En todo caso, el valor sociohistórico y cultural de la revista académica es y seguirá siendo decisivo para la universidad; un elemento eje para no olvidar de dónde venimos, lo mucho que hemos arado y los buenos frutos que hoy recogemos, gracias a la visión, al esfuerzo y a la conjunción de voluntades que hacen posible nuestros haceres y quehaceres.

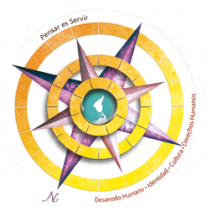




\section{Referencias}

Comisión Ad-hoc (1973). Acta 93. Universidad Nacional, Costa Rica. (1974). Acta 124. Universidad Nacional, Costa Rica.

EUNA (1982). Actas del Consejo Editorial. Universidad Nacional, Costa Rica

Erlicher, H. Ribler-Pika N (eds). 2014. Almacenes de un tiempo en fuga. Revistas culturales en la modernidad hispanoamericana. Consultado de: https://www.revistas-culturales.de/ es/buchseite/hanno-ehrlicher-nanette-ri\%C3\%9Fler-pipka-eds-almacenes-de-un-tiempo-en-fuga-revistas

Oliva, M. (2010). Los avatares de la Revista Repertorio Americano. Cuadernos Prometeo. Costa Rica: Universidad Nacional.

Piba, A. (1979). Editorial. Revista de .Ciencias Veterinarias, No. 1, sept. 1979

Pita, A. (2005). Revistas culturales y redes intelectuales Revistas culturales y redes intelectuales: una aproximación metodológica. Temas De Nuestra América. Revista De Estudios Latinoamericanos, 29(54), 177-194. Recuperado a partir de https://www. revistas.una.ac.cr/index.php/tdna/ article/view/6338

Sarlo, B. (1992). Intelectuales y revistas : razones de una práctica. In: América : Cahiers du CRICCAL, n 9-10, 1992. Le discours culturel dans les revues latino-américaines, 1940-1970. pp. 9-16. DOI : https://doi.org/10.3406/ ameri.1992.1047 